\title{
Faithful group actions on rooted trees induced by actions of quotients
}

\author{
Yaroslav Lavrenyuk, Volodymyr Mazorchuk, Andriy Oliynyk, \\ and Vitaliy Sushchansky
}

\begin{abstract}
We develop some new techniques of constructing (finite state) actions on homogeneous trees and apply them to various groups. In particular we show that there is a faithful action of the amalgameted free product $\mathbb{Z} * \mathbb{Z}$ on a rooted homogeneous tree of finite degree, described by finite state automorphisms.
\end{abstract}

\section{Introduction and preliminaries}

The class of all countable residually finite groups can be also characterized as the class of countable groups, which admit faithful actions on locally finite rooted trees. This class of groups plays an important role in the applications of the modern group theory to analysis, geometry, probability, fractal theory, etc. It contains groups with many extraordinary properties, in particular, Burnside type groups (i.e. infinite finitely generated torsion groups), groups of intermediate growth, amenable but non elementary amenable groups, groups of finite width, an the so-called just infinite groups. We refer the reader to the surveys [GNS], [Gr] for more details.

A natural subclass of residually finite groups is formed by the so-called finitely automatic groups, that is groups, whose elements act on a rooted homogeneous tree via the action, which can be described by finite automata. Many residually finite groups, for example free groups, free products of finite groups, linear groups $G L(n, \mathbb{Z}), n \geq 1$, etc., are known to be finitely automatic (see [BS], [GNS], [N], [Ol2]). Nevertheless, this class of groups is especially difficult to describe and there is still no reasonable criterion for a group to be finitely automatic. For many interesting groups the question of being finitely automatic is still open.

In this paper we develop some new techniques of constructing (finite state) actions of residually finite groups on homogeneous trees and apply them to various groups. Using these techniques in Section 2 we show that the amalgamated free product $\mathbb{Z} *_{\mathbb{Z}} \mathbb{Z}$ has a faithful finitely automatic action on a rooted homogeneous tree. Note, that this is the first explicit construction of a faithful finitely automatic action of an amalgameted free product, which is not a free product, on a regular rooted tree. As a corollary we obtain that the 


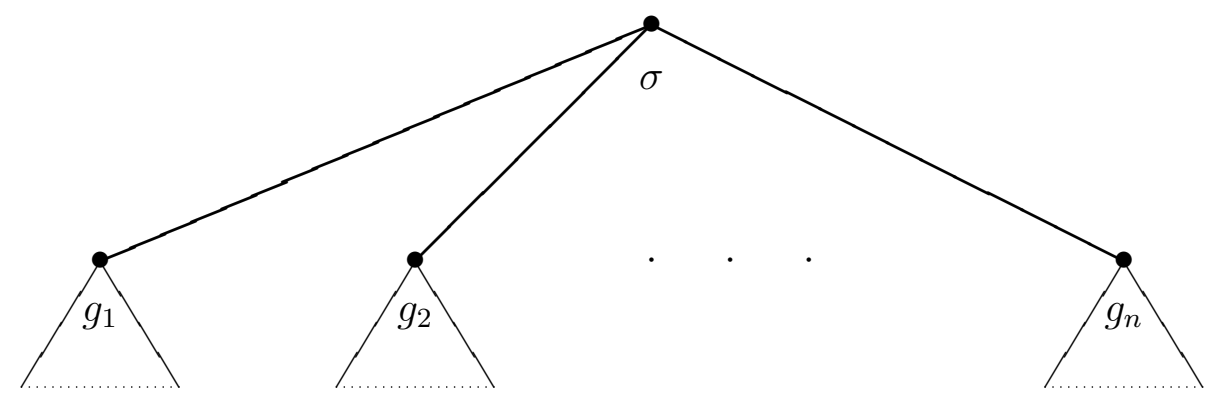

Figure 1: The automorphism $g=\left(g_{1}, \ldots, g_{n}\right) \sigma$

braid group $B_{3}$ has a faithful finitely automatic action on a regular rooted tree of degree 3 .

We show explicitly that the free product of free abelian groups of finite rank is recursively automatic (which means that the construction we present although does not give finite automata, but rather infinite automata with recursively described sets of states).

Trying to understand how one can extend a faithful finitely automatic action of a quotient (or quotients) to a faithful finitely automatic action of the original group and vice versa, we naturally come to the notion of almost identity elements in finitely generated groups. We introduce and study this notion in Section 3. In Section 4 we construct an action of the free power $\mathbb{Z}_{2}^{* n}$ on a rooted homogeneous tree of degree $n+1$ such that all automata, which define the action of the canonical generators of $\mathbb{Z}_{2}^{* n}$, have exactly two states. This result can be considered as a "dual" to the one obtained in [Ol1].

In the sequel we denote by $\mathcal{T}_{n}$ a rooted homogeneous tree of degree $n$ and by Aut $\mathcal{T}_{n}$ its automorphism group. For $\sigma \in S_{n}$ and $g_{1}, \ldots, g_{n} \in$ Aut $\mathcal{T}_{n}$ we denote by $\left(g_{1}, \ldots, g_{n}\right) \sigma$ the element of Aut $\mathcal{T}_{n}$, which acts as $\sigma$ on the edges adjacent to the root and as $g_{1}, \ldots, g_{n}$ on the subtrees initiated on the first level (see Figure 1). Note that each automorphism $g \in$ Aut $\mathcal{T}_{n}$ can be uniquely expressed in this form. We call automorphisms $g_{1}, g_{2}, \ldots, g_{n}$ the states of the first level of $g$ and $\sigma$ the rooted permutation of $g$. The states of the second level of $g$ are the states of the first level of the automorphisms $g_{1}, g_{2}, \ldots, g_{n}$. Inductively we define the states of the $n$-th level of $g$ for every positive integer $n$. The state of the 0 -th level of $g$ is $g$ itself. An automorphism, $g \in$ Aut $\mathcal{T}_{n}$, is called a finite state automorphism if $g$ has only a finite number of different states. According to S. Sidki [S], an automorphism, $g \in$ Aut $\mathcal{T}_{n}$, is called recursive if the set of all its states is contained in some finitely generated subgroup $G$ of Aut $\mathcal{T}_{n}$. All finite state automorphisms and all recursive automorphisms form in Aut $\mathcal{T}_{n}$ the subgroups $F A_{n}$ and $R A_{n}$ respectively. We obviously have $F A_{n}<R A_{n}$. Subgroups of $F A_{n}$ are called finitely automatic and subgroups of $R A_{n}$ are called recursively automatic. We always denote by $e$ the identity transformation. 


\section{Lifting the action of some quotients to the group action}

2.1. Let $G$ be a group and $H_{i}, i \in I$, be a finite or countable collection of normal subgroups of $G$. Set $H=\cap_{i \in I} H_{i}$ and $G_{i}=G / H_{i}$. The following lemma is an easy observation.

Lemma 1. 1. Assume that for every $i \in I$ the group $G_{i}$ acts faithfully on $\mathcal{T}_{n}$. Then $G / H$ acts faithfully on $\mathcal{T}_{n}$.

2. Let $I$ be finite. Assume that for every $i \in I$ the group $G_{i}$ acts faithfully on $\mathcal{T}_{n}$ by finite state automorphisms. Then $G / H$ acts faithfully on $\mathcal{T}_{n}$ by finite state automorphisms.

Proof. Let $\varphi_{i}: G_{i} \rightarrow$ Aut $\mathcal{T}_{n}$ be some monomorphisms, $p_{i}: G \rightarrow G_{i}$ be the canonical epimorphism, and $f: I \rightarrow \mathbb{N}$ be any injection. For $g \in G$ we construct the element $\bar{g} \in$ Aut $\mathcal{T}_{n}$ using the following recursion: $\bar{g}=\left(\alpha_{1}, e, \ldots, e, \beta_{1}\right) e$, where for $j \in \mathbb{N}$ we have

$$
\beta_{j}=\left\{\begin{array}{ll}
e, & j \notin f(I) \\
\varphi_{i}(g), & j=f(i),
\end{array} \quad \alpha_{j}=\left(\alpha_{j+1}, e, \ldots, e, \beta_{j+1}\right) e .\right.
$$

It is straightforward that $g \mapsto \bar{g}$ is a homomorphism and that $\bar{h}=e$ for any $h \in H$. Conversely, if $g \in G \backslash H$, then $\varphi_{i}(g) \neq e$ for some $i \in I$ and thus $\bar{g} \neq e$. Hence the kernel of our homomorphism is exactly $H$ and the first statement is proved.

To prove the second statement it is enough to observe that, by construction, the cardinality of the set of all states of $\bar{g}$ does not exceed $n^{2 m}\left(\sum_{i \in I} k_{i}+|I|+1\right)$, where $k_{i}$ denotes the number of states of $\varphi_{i}(g)$ and $m=\max _{i} f(i)$. Hence if $I$ is finite, and all $\varphi_{i}(g)$ are finite state, $\bar{g}$ is finite state as well.

Remark 2. Define the quotient $\bar{G}\left(\left\{H_{i}, i \in I\right\}\right)$ of $G$ as the image of the homomorphism $\psi: G \rightarrow \prod_{i \in I} G / H_{i}$, where $\psi(g)=\left(\left\{g H_{i}, i \in I\right\}\right)$ for any $g \in G$. By Remak's Theorem the quotients $G / H$ and $\bar{G}\left(\left\{H_{i}, i \in I\right\}\right)$ are canonically isomorphic.

We would like to propose a construction, which allows one to lift the action of the quotient over the center to the action of the whole group. Let $G$ be a group. Assume that the center $Z(G)$ of $G$ contains the direct product $Z_{1} \times Z_{2}$ of its proper subgroups $Z_{1}$ and $Z_{2}$. Denote by $N_{i}$ the quotient $G / Z_{i}, i=1,2$. As above, consider the quotient $\bar{G}\left(Z_{1}, Z_{2}\right)$ of $G$, consisting of all pairs $\left(g Z_{1}, g Z_{2}\right), g \in G$. By Remark 2 this group is canonically isomorphic to $G$. Therefore, by Lemma 1 we have the following:

Lemma 3. If for some $n \in \mathbb{N}$ the quotients $N_{1}$ and $N_{2}$ of $G$ act faithfully on the homogeneous rooted tree $\mathcal{T}_{n}$ by (finite state) automorphisms then the same is true for the group $G$.

Fix now the group $G$ such that the center $Z(G)$ is a cyclic group which is not decomposable into a direct product of proper subgroups. Then we have $Z(G)=\langle a\rangle$ and the order $\operatorname{ord}(a)$ of $a$ is either infinite or a power of a prime. Denote by $N$ the quotient $G / Z(G)$. 
Call an epimorphism, $\phi$, from $G$ onto some nontrivial group $M$ reducing if $\phi(c) \neq e$ for all $c \in Z(G), c \neq e$. This is equivalent to the requirement $\operatorname{Ker}(\varphi) \cap Z(G)=\{e\}$. Then by Lemma 1 we have:

Lemma 4. Let $\phi: G \rightarrow M$ be a reducing epimorphism. Assume that for some $n$ both $N$ and $M$ admit a faithful representation by (finite state) automorphisms of the homogeneous rooted tree $\mathcal{T}_{n}$. Then $G$ admits a faithful representation by (finite state) automorphisms of $\mathcal{T}_{n}$.

Now, additionally, suppose that $G$ is finitely generated. We are going to formulate a criterion of the existence for some special reducing epimorphism for $G$.

Let $G$ have presentation $\left\langle x_{1}, \ldots, x_{k} \mid w_{i}\left(x_{1}, \ldots, x_{k}\right), i \geq 1\right\rangle$ and assume that $Z(G)=$ $\langle a\rangle$, where $a=w_{0}\left(x_{1}, \ldots, x_{k}\right)$.

Denote by $c_{j}^{(i)}$ the sum of the degrees of the letter $x_{j}$ in the word $w_{i}\left(x_{1}, \ldots, x_{k}\right)$, where $i \geq 0$ and $1 \leq j \leq k$. For each $i \geq 0$ define the linear form

$$
l_{i}\left(z_{1}, \ldots, z_{k}\right)=c_{1}^{(i)} z_{1}+\ldots+c_{k}^{(i)} z_{k}
$$

over $z_{1}, \ldots, z_{k}$.

Consider the following system of linear equations and inequalities

$$
\begin{aligned}
& l_{i}\left(z_{1}, \ldots, z_{k}\right)=0, \quad i \geq 1 \\
& l_{0}\left(z_{1}, \ldots, z_{k}\right) \neq 0 .
\end{aligned}
$$

We consider (1) over the set $\mathbb{Z}^{+}$of all non-negative integers or over the set $\mathbb{Z}_{p^{s}}$ of reminders modulo $p^{s}$ for some prime $p$ and $s \geq 1$.

Lemma 5. 1. Let $Z(G) \simeq \mathbb{Z}$. Then there exists a reducing epimorphism from $G$ onto $\mathbb{Z}$ if and only if the system (1) admits a non-negative integral solution.

2. Let $Z(G) \simeq \mathbb{Z}_{p^{s}}$. Then a reducing epimorphism from $G$ onto some nontrivial subgroup of $\mathbb{Z}_{p^{s}}$ exists if and only if the system (1) admits a solution over $\mathbb{Z}_{p^{s}}$.

Proof. Let $\left(z_{1}^{0}, \ldots, z_{k}^{0}\right)$ be a solution to (1). Then the map

$$
x_{j} \rightarrow a^{z_{j}^{0}}, j=1, \ldots, k,
$$

can be extended to a homomorphism, $\phi: G \rightarrow Z(G)$, since $w_{i}\left(a^{z_{1}^{0}}, \ldots, a^{z_{k}^{0}}\right)=1$ for $i \geq 1$. We also have

$$
\phi(a)=\phi\left(w_{0}\left(x_{1}, \ldots, x_{k}\right)\right)=a^{l_{0}\left(z_{1}^{0}, \ldots, z_{k}^{0}\right)} \neq 1 .
$$

Then $\phi(G)$ is a nontrivial subgroup of $Z(G)$, which implies the required statement in both cases.

On the other hand, let $\phi: G \rightarrow Z(G)$ be some reducing epimorphism. Then $\phi_{a}\left(x_{j}\right)=$ $a^{z_{j}^{0}}$ for some $0 \leq z_{j}^{0}<\operatorname{ord}(a), 1 \leq j \leq k$. Since $\phi$ is an epimorphism, we automatically have $\phi\left(w_{i}\left(x_{1}, \ldots, x_{k}\right)\right)=1$ and $a^{l_{i}\left(z_{1}^{0}, \ldots, z_{k}^{0}\right)}=1, i \geq 1$. Further, the fact that $\phi$ is reducing implies (2). Thus $\left(z_{1}^{0}, \ldots, z_{k}^{0}\right)$ is a non-trivial solution to (1). This completes the proof. 
2.2. Now we can apply the statements of the previous subsection in the following situation:

Theorem 6. Let $k, l \geq 2$. Then the amalgamated free product

$$
G=\mathbb{Z} *_{\mathbb{Z}} \mathbb{Z}=\left\langle x_{1}, x_{2} \mid x_{1}^{k}=x_{2}^{l}\right\rangle
$$

acts faithfully on the homogeneous rooted tree $\mathcal{T}_{n}, n=\max \{k, l\}$, by finite state automorphisms.

Proof. The center $Z(G)$ of $G$ is the infinite cyclic group, generated by $x_{1}^{k}$ (see for example [MKS, Corollary 4.5, p.211]). Let $\bar{x}_{i}=x_{i} Z(G), i=1,2$. Then the quotient $G / Z(G)$ has the following presentation:

$$
G / Z(G)=\left\langle\bar{x}_{1}, \bar{x}_{2} \mid \bar{x}_{1}^{k}=\bar{x}_{2}^{l}=1\right\rangle .
$$

The group $G / Z(G)$ is isomorphic to the free product $\mathbb{Z}_{n} * \mathbb{Z}_{l}$ of finite cyclic groups. By [Ol2], this quotient acts faithfully by finite state automorphisms on the homogeneous rooted tree $\mathcal{T}_{n}$ of degree $n=\max \{k, l\}$.

The system (1) for $G$ has the form

$$
\begin{aligned}
& k z_{1}-l z_{2}=0 \\
& k z_{1} \neq 0 .
\end{aligned}
$$

This system has the integral solution $(l, k)$. Therefore, by Lemmas 4 and 5 , the group $G$ acts faithfully on $\mathcal{T}_{n}$ by finite state automorphisms. The proof is complete.

Remark 7. We have shown that there is an epimorphism from $G$ onto $Z(G)$ (the reducing epimorphism). We can even write the presentation for $Z(G)$ in terms of the generators of $G$ explicitly:

$$
Z(G)=\left\langle x_{1}, x_{2} \mid x_{1}^{t}=x_{2}^{s}, x_{1} x_{2}=x_{2} x_{1}\right\rangle \simeq \mathbb{Z},
$$

where $t=\frac{k}{g c d(k, l)}$ and $s=\frac{l}{g c d(k, l)}$. A generator, a, of this cyclic group can be chosen as the product $x_{1}^{d} x_{2}^{c}$ where $\operatorname{gcd}(k, l)=c k+d l$.

Remark 8. Let $H$ be the kernel of the reducing epimorphism of $G$ onto $Z(G)$. Then the images of the generators $x_{1}$ and $x_{2}$ of $G$ under the isomorphism $\psi: G \rightarrow \bar{G}(Z(G), H)$ are

$$
\psi\left(x_{1}\right)=\left(\bar{x}_{1}, a^{s}\right), \quad \psi\left(x_{2}\right)=\left(\bar{x}_{2}, a^{t}\right),
$$

where $a, s, t$ are defined above.

Recall that Artin's braid group $B_{n}$ has the following presentation in terms of generators and relations (see, for example, $[\mathrm{Bi}]$ )

$$
B_{n}=\left\langle\sigma_{1}, \ldots, \sigma_{n-1}\left|\sigma_{i} \sigma_{j}=\sigma_{j} \sigma_{i},\right| i-j \mid>1 ; \sigma_{i} \sigma_{i+1} \sigma_{i}=\sigma_{i+1} \sigma_{i} \sigma_{i+1}, i=1, \ldots, n-1\right\rangle .
$$

We immediately obtain 
Corollary 9. The braid group $B_{3}$ acts faithfully on the homogeneous rooted tree $\mathcal{T}_{3}$ by finite state automorphisms.

Proof. It is well-known (see, for example, [Bi]) that the braid group $B_{3}$ is isomorphic to the amalgamated free product

$$
\mathbb{Z} *_{\mathbb{Z}} \mathbb{Z} \simeq\left\langle x_{1}, x_{2} \mid x_{1}^{3}=x_{2}^{2}\right\rangle .
$$

The standard generators of $B_{3}$ in this presentation are $\sigma_{1}=x_{1}^{-1} x_{2}$ and $\sigma_{2}=x_{2}^{-1} x_{1}^{2}$. The assertion of the theorem now follows from Theorem 6 .

Now we describe one possible construction of automata, which correspond to the generators $x_{1}, x_{2}$ of $B_{3}$. Let $a$ be the generator of $Z\left(B_{3}\right)$ and

$$
B_{3} / Z\left(B_{3}\right)=\left\langle\bar{x}_{1}, \bar{x}_{2} \mid \bar{x}_{1}^{3}=\bar{x}_{2}^{2}=e\right\rangle \simeq \mathbb{Z}_{3} * \mathbb{Z}_{2} .
$$

Assume that we are given a faithful action of $Z\left(B_{3}\right)$ and $B_{3} / Z\left(B_{3}\right)$ on $\mathcal{T}_{3}$ by finite state automorphisms. Define the following automorphisms of $\mathcal{T}_{3}$ :

$$
a_{1}=\left(\bar{x}_{1}, a^{2}, e\right), \quad a_{2}=\left(\bar{x}_{2}, a^{3}, e\right) .
$$

By Remark 7 the automorphisms $a_{1}$ and $a_{2}$ generate $B_{3}$. Now, using some known constructions, one can construct two finite automata, defining the automorphisms $a_{1}$ and $a_{2}$. For instance, if we choose the automata from [Ol2] as the generators of $\mathbb{Z}_{2} * \mathbb{Z}_{3}$ and the automaton, realizing the so-called "adding machine" (see e.g. [GNS]), as the generator of $\mathbb{Z}$, we obtain two automata for $a_{1}$ and $a_{2}$ with 14 and 13 states respectively.

Let $p$ be a prime. Recall that a group, $G$, is called a residually finite p-group if for every its non-indentity element $x$ there exists a finite $p$-group, $G_{x}$, and a homomorphism, $\varphi_{x}: G \rightarrow G_{x}$, such that $\varphi_{x}(x) \neq e$. We leave without proof the following simple and well-known fact.

Lemma 10. Every countable residually finite p-group acts faithfully on the tree $\mathcal{T}_{p}$.

Being a subgroup of the automorphism group of a free group of the corresponding rank the braid group $B_{n}$ is residually finite. It is then easy to see that $B_{n}$ acts faithfully on a rooted homogeneous tree of finite degree. Let us show that $B_{n}$ acts faithfully on a rooted homogeneous tree of degree $n$. The easiest way to show this uses the results on the lower central series for the pure braid group $P B_{n}$ from $[\mathrm{FR}]$.

Proposition 11. The braid group $B_{n}$ acts faithfully on the tree $\mathcal{T}_{n}$.

Proof. The braid group $B_{n}$ contains the pure braid group $P B_{n}$ as a normal subgroup. The corresponding quotient is isomorphic to the symmetric group $S_{n}$. Since $S_{n}$ acts faithfully on $\mathcal{T}_{n}$ in the natural way (permuting the edges adjacent to the root), it is sufficient to prove that the group $P B_{n}$ acts faithfully on the binary tree $\mathcal{T}_{2}$. Note, that the quotients of the lower central series of $P B_{n}$ are free abelian groups of finite rank and the intersection 
of the components of this series is trivial (see [FR]). For an arbitrary non-trivial $x \in P B_{n}$ consider a term, $\Gamma_{m}$, of the lower central series for $P B_{n}$ such that $x \notin \Gamma_{m}$. The quotient group $P B_{n} / \Gamma_{m}$ is a finitely generated nilpotent torsion-free group. Hence it is a residually finite $p$-group for each prime $p$ (see $[\mathrm{R}]$ ). Therefore the group $P B_{n}$ is a residually finite 2-group and the proof is completed by applying Lemma 10.

After Propsotion 11 the following natural problem arises:

Problem 12. Does there exist a faithful action of the braid group $B_{n}, n \geq 4$, on a rooted homogeneous tree by finite state automorphisms?

2.3 The free product $G=\mathbb{Z}^{n} * \mathbb{Z}^{l}$ has the presentation

$$
G=\left\langle x_{1}, \cdots, x_{n}, y_{1}, \cdots, y_{l} \mid R\right\rangle,
$$

where $R$ is the set of the following relations:

$$
\begin{aligned}
& x_{i} x_{j}=x_{j} x_{i}, 1 \leq i<j \leq n \\
& y_{s} y_{t}=y_{t} y_{s}, 1 \leq s<t \leq l .
\end{aligned}
$$

For every $k \in \mathbb{N}$ define

$$
\begin{aligned}
& R_{k}^{(x)}=\left\{x_{i}=x_{1}^{i^{k}} \mid 1 \leq i \leq n\right\}, \\
& R_{k}^{(y)}=\left\{y_{j}=y_{1}^{j^{k}} \mid 1 \leq j \leq l\right\},
\end{aligned}
$$

and denote by $N_{k}$ the group

$$
N_{k}=\left\langle x_{1}, \cdots, x_{n}, y_{1}, \cdots, y_{l} \mid R, R_{k}^{(x)}, R_{k}^{(y)}\right\rangle .
$$

Lemma 13. For every $k \in \mathbb{N}$ the group $N_{k}$ is a free group of rank 2 with free generators $x_{1}$ and $y_{1}$.

Proof. That $x_{1}$ and $y_{1}$ generate $N_{k}$ follows from the relations $R_{k}^{(x)}$ and $R_{k}^{(y)}$. That there are no relations on $x_{1}$ and $y_{1}$ is straightforward.

Dyck's Theorem implies that for every $k \in \mathbb{N}$ the group $N_{k}$ is the quotient of $G$ under the homomorphism $\psi_{k}$ defined as follows: $\psi_{k}\left(x_{i}\right)=x_{i}$ and $\psi_{k}\left(y_{j}\right)=y_{j}(1 \leq i \leq n, 1 \leq j \leq l)$. Denote by $H_{k}$ the kernel of $\psi_{k}$ and let $H$ be the intersection $\cap_{k \geq 1} H_{k}$.

Lemma 14. The group $H$ is trivial.

Proof. Each element in $G$ can be represented as a word,

$$
w=w_{11}\left(x_{1}, \ldots, x_{n}\right) w_{12}\left(y_{1}, \ldots, y_{l}\right) \ldots w_{m 1}\left(x_{1}, \ldots, x_{n}\right) w_{m 2}\left(y_{1}, \ldots, y_{l}\right),
$$

where $w_{j i}$ are nontrivial words for all $1 \leq j \leq m, i=1,2$, except, possibly, for $w_{11}$ and $w_{m 2}$. Let $w \in H$ and assume additionally that $w \neq e$. Since each quotient $N_{k}$ is free of rank 2 with generators $\psi_{k}\left(x_{1}\right)=x_{1}$ and $\psi_{k}\left(y_{1}\right)=y_{1}$, then $w \in H_{k}, w \neq e$, implies that there exists 
$j, 1 \leq j \leq m$, such that either $w_{j 1}\left(x_{1}, \ldots, x_{n}\right) \in H_{k} \backslash\{e\}$ or $w_{j 2}\left(y_{1}, \ldots, y_{l}\right) \in H_{k} \backslash\{e\}$. Hence there exist $j_{0}$ and $i_{0}\left(1 \leq j_{0} \leq m, i_{0} \in\{1,2\}\right)$ such that $w_{j_{0} i_{0}}$ is non-trivial and belongs to $H_{k}$ for infinitely many $k \in \mathbb{N}$. Let $k_{1}, k_{2}, \ldots\left(k_{1}<k_{2}<\ldots\right)$ be the sequence of such $k$. Without loss of generality we may assume that $i_{0}=1$. For some $u_{1}, \ldots, u_{n} \in \mathbb{Z}$ we have

$$
w_{j_{0} 1}\left(x_{1}, \ldots, x_{n}\right)=x_{1}^{u_{1}} \ldots x_{n}^{u_{n}}
$$

as $w_{j_{0} 1}$ is an element from the free abelian group $\mathbb{Z}^{n}$.

The conditions $w_{j_{0} 1} \in H_{k_{s}}, s \geq 1$, mean that $\left(u_{1}, \ldots, u_{n}\right)$ is a solution to the infinite homogeneous system of linear equations

$$
z_{1}+2^{k_{s}} z_{2}+\ldots+n^{k_{s}} z_{n}=0, s \geq 1 .
$$

The matrix of this system has an $n \times n$-minor, which is the generalized Vandermonde $n \times n$-determinant (see for instance [Ga]). This implies that the system (4) has only the trivial solution and we get a contradiction. The statement follows.

Theorem 15. The free product $\mathbb{Z}^{n} * \mathbb{Z}^{l}$ acts faithfully on $\mathcal{T}_{2}$ by recursively automatic automorphisms.

Proof. The free product $G=\mathbb{Z}^{n} * \mathbb{Z}^{l}$ is isomorphic to the quotient $G / H$ by Lemma 14 . From Lemma 13 and [Ol1] it follows that for every $k \in \mathbb{N}$ the group $N_{k}$ has a faithful representation by finite state automorphisms of the binary rooted tree $\mathcal{T}_{2}$. Hence by Lemma 1 the quotient $G / H$ has a faithful representation by automorphisms of $\mathcal{T}_{2}$. An explicit construction can be given as follows. Let $a$ and $b$ be finite state automorphisms of $\mathcal{T}_{2}$, which generate the free group of rank 2. By construction from the proof of Lemma 1 the subgroup of $A u t \mathcal{T}_{2}$ generated by

$$
\begin{aligned}
& \bar{x}_{i}=\left(\bar{x}_{i}^{i}, a\right), 1 \leq i \leq n ; \\
& \bar{y}_{j}=\left(\bar{y}_{j}^{j}, b\right), \quad 1 \leq j \leq l,
\end{aligned}
$$

is isomorphic to $G / H$. It is obviously recursively automatic.

It was shown in $[\mathrm{CP}]$ that the subgroup $L_{n}$ of $B_{n}$, generated by $\lambda_{i}=\sigma_{i}^{2}, i=1, \ldots, n-1$, has the following presentation:

$$
L_{n}=\left\langle\lambda_{1}, \ldots, \lambda_{n-1}\left|\lambda_{i} \lambda_{j}=\lambda_{j} \lambda_{i},\right| i-j \mid>1\right\rangle .
$$

In particular, the subgroup $L_{4}$ of $B_{4}$ has the presentation

$$
L_{4}=\left\langle x_{1}, x_{2}, y \mid x_{1} x_{2}=x_{2} x_{1}\right\rangle
$$

i.e. it is isomorphic to the free product $\mathbb{Z}^{2} * \mathbb{Z}$. Applying the embedding from the last theorem in this partial case we obtain an embedding of $L_{4}$ into Aut $\mathcal{T}_{2}$.

Corollary 16. The following automorphisms of $\mathcal{T}_{2}$ :

$$
\bar{x}_{1}=\left(\bar{x}_{1}, a\right), \quad \bar{x}_{2}=\left(\bar{x}_{2}^{2}, a\right), \quad \bar{y}=(\bar{y}, b),
$$

generate a group isomorphic to $L_{4}$.

Note that both $\bar{x}_{1}$ and $\bar{y}$ are finite state automorphisms. The above result motivates the following natural question: Do $L_{n}, n \geq 5$, admit recursively automatic faithful actions on $\mathcal{T}_{n}$ ? 


\section{Techniques of almost identity elements}

In this section we use the ideas of Section 2 to define and investigate certain elements, which we call almost identity elements, in free products and extend this notion to all groups. In case of free products it also leads to a pulling back a faithful action of a free product on a rooted homogeneous tree to a faithful action of the quotient of this free product modulo the group of almost identity elements.

3.1. Let $G_{1}, \ldots, G_{k}$ be groups and $G=G_{1} * \cdots * G_{k}$. For $i=1, \ldots, k$ denote by $H_{i}$ the normal closure of the canonical copy of $G_{i}$ inside $G$, that is the kernel of the natural surjection $G \rightarrow G_{1} * \cdots * G_{i-1} * G_{i+1} * \cdots * G_{k}$. We set $A=A\left(G_{1}, \ldots, G_{k}\right)=H_{1} \cap \cdots \cap H_{k}$ and call the elements of $A$ almost identity elements. Alternatively, one can describe the elements of $A$ in terms of the following deletion process. For each $i=1, \ldots, k$ choose a set $\left\{g_{j}^{(i)} \mid j \in J_{i}\right\}$ of generators for $G_{i}$, and for every word $w=w\left(g_{j}^{(i)}\right), i \in\{1, \ldots, k\}$ and $j \in J_{i}$, denote by $\mathfrak{d}_{i}$ the operation of deleting in $w$ all occurrences of all $g_{j}^{(i)}, j \in J_{i}$, and their inverses.

Lemma 17. Let $h \in G$. Then the following conditions are equivalent.

(i) $h$ is an almost identity element;

(ii) there exists a word, $w=w\left(g_{j}^{(i)}\right)$, such that $w=h$ and $\mathfrak{d}_{i}(w)=e$ in $G$ for all $i$;

(iii) for all words $w=w\left(g_{j}^{(i)}\right)$ such that $w=h$ we have $\mathfrak{d}_{i}(w)=e$ in $G$ for all $i$.

Proof. (i) $\Rightarrow$ (iii) follows from the definition of $A$ and (iii) $\Rightarrow$ (ii) is obvious. Finally (ii) $\Rightarrow(\mathrm{i})$ follows from the fact that $G$ is a free product of $G_{1}, \ldots, G_{k}$.

Under the assumptions above, the quotient $G / A$ is big enough. In particular, this quotient surjects onto each $G_{1} * \cdots * G_{i-1} * G_{i+1} * \cdots * G_{k}$, which is equivalent to saying that every element in $H$ must contain generators from every $G_{i}$ in any word, representing this element.

The second condition of Lemma 17 allows one to define almost identity elements with respect to a fixed (finite) system of generators for any finitely generate group $G$ in a straightforward way. Let $G$ be a group, generated by $\mathbf{g}=\left\{g_{1}, \ldots, g_{k}\right\}$. For a word, $w$, in $g_{1}, \ldots, g_{k}$ and $i \in\{1, \ldots, k\}$ let $\mathfrak{d}_{i}$ denote the operation of deleting all occurrences of $g_{i}^{ \pm 1}$ in w. An element, $g \in G$, will be called almost identity with respect to $\mathrm{g}$ provided that there exists a word, $w$, in $g_{1}, \ldots, g_{k}$ such that $w$ represents $g$ and $\mathfrak{d}_{i}(w)=e$ for all $i$. It is clear that in this general case the second and the third conditions of Lemma 17 are not equivalent. Neither one can in the general case reformulate the second condition in any way similar to the first condition. Remark that the almost identity elements in a free product and with respect to a system of generators in this free product coincide only if all $G_{i}$ are cyclic groups with the chosen generators being generators of $G_{i}$.

Lemma 18. Let $G$ be a group, generated by $\mathbf{g}=\left\{g_{1}, \ldots, g_{k}\right\}$, and $A=A(G, \mathbf{g})$ be the corresponding set of almost identity elements. Then $A$ is a normal subgroup of $G$. 
Proof. It is clear that $A$ is a subgroup and that conjugating an almost identity element gives an almost identity element. The statement follows.

Remark 19. Let $G$ be a group, $\mathbf{g}=\left\{g_{1}, \ldots, g_{k}\right\}$ be a finite system of generators of $G$, and $w$ be an almost identity word with respect to $\mathbf{g}$ in $G$ (i.e. $\mathfrak{d}_{i}(w)=e$ for all $\left.i\right)$. In the case of a free product of groups as discussed above it is easy to see that $\mathfrak{d}_{i} \circ \mathfrak{d}_{j}(w)=e$ for all $i \neq j$. In general this is not true.

3.2. It seems to be interesting to try to find the subgroups of almost identity elements in known groups, especially, those which are close to simple groups. Here we give a collection of basic statement about almost identity elements and formulate several questions.

We start with the description of the groups of almost identity elements for the finitely generated abelian groups.

Lemma 20. Let $G$ be a finitely generated abelian group, $\mathbf{g}=\left\{g_{1}, \ldots, g_{k}\right\}$ be a system of generators for $G$, and $A=A(G, \mathbf{g})$. Then

$$
A=\left(\bigcap_{i=1}^{k}\left\langle g_{i}\right\rangle\right) \bigcap\{y \in G \mid(k-1) y=e\} .
$$

In particular, $A$ is always cyclic, and $A=G$ if and only if $k=1$.

Proof. That $A=G$ if $k=1$ is clear. Observe that some $x \in G, x=g_{1}^{a_{1}} \ldots g_{k}^{a_{k}}$, belongs to $A$ if and only if $x g_{i}^{-a_{i}}=e$ for all $i$. That is $x=g_{i}^{a_{i}}$ for all $i$, and $\prod_{j \neq i} g_{j}^{a_{j}}=x^{k-1}=e$ for all $j$. This implies (7). Further, that $A$ is cyclic follows from the fact that it is a subgroup of $\left\langle g_{1}\right\rangle$ by (7). In particular, $A \neq G$ if $G$ is not cyclic. Finally, let us assume that $G=\langle t\rangle$ and $k>1$. If $G$ is infinite, then $(k-1) t \neq e$ and thus $t \notin A$ by (7). If $G$ is finite, say $|G|=n$, then $k \leq n$ and thus $k-1<n$, implying $(k-1) t \neq e$ and hence $t \notin A$ by $(7)$ as well.

Lemma 21. Let $G$ be a group, $\mathbf{g}=\left\{g_{1}, \ldots, g_{k}\right\}$ be a system of generators for $G, A=$ $A(G, \mathbf{g})$, and $N$ be a normal subgroup of $G$. Assume that $g_{i} N \neq g_{j} N$ for all $i \neq j$. Then the natural surjection $p: G \rightarrow G / N$ induces a map $\bar{p}: A(G, \mathbf{g}) \rightarrow A\left(G / N,\left\{g_{1} N, \ldots, g_{k} N\right\}\right)$.

Proof. From the definition of an almost identity element it follows that this property is preserved under epimorphisms and with respect to the image of the generating set.

After Lemma 21 a natural question is: When is $\bar{p}$ surjective?

We proceed by listing some properties of $A(G, \mathbf{g})$ in the case, when the generating set has two elements.

Proposition 22. Let $G$ be a group, generated by $\mathbf{g}=\left\{g_{1}, g_{2}\right\}$, and $A=A(G, \mathbf{g})$. Then $A=[G, G]$. 
Proof. We start with $[G, G] \subset A$. For $x, y \in G$ the inclusion $[x, y] \in A$ follows directly from the definition of an almost identity element, applied to any words, representing $x$ and $y$ in terms of $g_{1}$ and $g_{2}$. Therefore $[G, G] \subset A$.

To complete the proof we have to show that $A \subset[G, G]$. Consider the canonical projection $p: G \rightarrow G /[G, G]$. Because of Lemma 21, to prove $A \subset[G, G]$ it is enough to show that $A\left(G /[G, G],\left\{g_{1}[G, G], g_{2}[G, G]\right\}\right)=\{e\}$. If $g_{1}[G, G] \neq g_{2}[G, G]$ the equality $A\left(G /[G, G],\left\{g_{1}[G, G], g_{2}[G, G]\right\}\right)=\{e\}$ follows from Lemma 20 since

$$
A\left(G /[G, G],\left\{g_{1}[G, G], g_{2}[G, G]\right\}\right) \subset\{y \in G /[G, G] \mid(2-1) y=e\}=\{e\} .
$$

Let us now assume that $g_{1}[G, G]=g_{2}[G, G]=x$ and that the order of $g_{1}[G, G]$ in $G /[G, G]$ is $n$. We are going to prove that $g_{1}^{i} \notin A$ for all $i<n$, which would also imply $A \subset[G, G]$. Let $w=w\left(g_{1}, g_{2}\right)$ be any word in $G$ such that $w=g_{1}^{i}$. Assume that $w$ becomes $e$ if we delete all occurrences of $g_{1}$. Then the sum of all exponents of $g_{2}$ in $w$ equals 0 . Analogously, if $w$ becomes $e$ if we delete all occurrences of $g_{2}$, then that the sum of all exponents of $g_{2}$ in $w$ equals 0 . Now let us consider the coset $w[G, G]$. Since $G /[G, G]$ is cyclic, we have $w[G, G]=x^{l}[G, G]$ for some $l$. Since the sums of all exponents of both $g_{1}$ and $g_{2}$ in $w$ are zero, it follows that $l=0$. On the other hand, $w=g_{1}^{i}$ implying $l=i<n$, which contradicts the fact that the order of $x$ is $n$. This completes the proof.

For 2-element generating sets we can formulate a sufficient condition for the map from Lemma 21 to be surjective in terms of relations.

Proposition 23. Let $G=\left\langle g_{1}, g_{2} \mid R\right\rangle$ be a group such that all relations in $R$ are homogeneous and $\mathbf{g}=\left\{g_{1}, g_{2}\right\}$. Let $N \triangleleft A(G, \mathbf{g})$. Assume that $g_{1} N$ and $g_{2} N$ are different elements of $G / N$. Then $A(G, \mathbf{g})$ surjects onto $A\left(G / N,\left\{g_{1} N, g_{2} N\right\}\right)$ under the canonical homomorphism $p: G \rightarrow G / N$.

Proof. It is easy to see that $\varphi(A(G, \mathbf{g})) \subset A\left(G / N,\left\{g_{1} N, g_{2} N\right\}\right)$. Take an element, $a \in$ $A\left(G / N,\left\{g_{1} N, g_{2} N\right\}\right)$, and let $w=w\left(g_{1} N, g_{2} N\right)$ be a word such that $a=w$. This means that, deleting all occurrences of $g_{i}, i=1,2$, from $w$, we will get an element in $N$. Let us fix $i=1,2$ and denote by $v$ the corresponding element, obtained by deleting all occurrences of $g_{i}$ in $w$. Thus we have $v=g_{3-i}^{s}$. Now let us recall that all words, equivalent to $v$ in $G$, are obtained from $v$ by a finite number of insertions and deletions of the relations in $G$. Since $v \in N \subset A(G, \mathbf{g})$, there exists a word, $\hat{v}=\hat{v}\left(g_{1}, g_{2}\right)$, such that $\hat{v}=v$, and deleting all occurrences of any of the generators from $\hat{v}$ gives an identity in $G$. This implies that the sum of all exponents of $g_{j}, j=1,2$, in $\hat{v}$ is zero. Therefore the same must be true also for $v$ since all relations in $G$ are homogeneous. This implies that $v=e$ and thus the sum of all exponents $g_{j}, j=1,2$, in $w$ is zero, which gives $w\left(g_{1}, g_{2}\right) \in A(G, \mathbf{g})$ and thus $w\left(g_{1} N, g_{2} N\right) \in A\left(G / N,\left\{g_{1} N, g_{2} N\right\}\right)$.

Remark that, under the assumptions of Proposition 23, and by the same arguments as in the proof of Proposition 23, one obtains that $g_{1} A(G, \mathbf{g}) \neq g_{2} A(G, \mathbf{g})$.

The above analysis motivates the following questions: Let $G$ be a group and $\mathbf{g}=$ $\left\{g_{1}, \ldots, g_{k}\right\}, k>1$, be a fixed system of generators in $G$, and $A=A(G, \mathbf{g})$. When 
$G=A$ ? Assume that the elements $\left\{g_{1} A, \ldots, g_{k} A\right\}$ are pairwise different in $G / A$. Is $A\left(G / A,\left\{g_{1} A, \ldots, g_{k} A\right\}\right)$ trivial?

In the above question it is not possible to take away the condition "the elements $\left\{g_{1} A, \ldots, g_{k} A\right\}$ are pairwise different in $G / A$ ". This will follows from the following example.

Example 24. $A\left(S_{n},\{(1,2), \ldots,(n-1, n)\}\right)=A_{n}$.

Proof. 1) Let $n=2 k+1>2$.

We set $\mathbf{g}=\{(1,2), \ldots,(n-1, n)\}$. Define the permutations $\tau_{i}, i=2, \ldots, n$, recursively as follows: $\tau_{2}=(1,2), \tau_{i}=\left[\tau_{i-1},(i-1, i)\right]$. It is easy to see that $\tau_{n} \in A\left(S_{n}, \mathbf{g}\right)$ and that all $\tau_{i}$ are non-trivial. In particular, $A\left(S_{n}, \mathbf{g}\right) \neq\{e\}$. On the other hand, odd permutations can not belong to $A\left(S_{n}, \mathbf{g}\right)$ by the following parity arguments: if $g \in S_{n}$ is odd and is written as a product of generators, then, since the number of generators is even, at least one generator occurs even number of times. It follows that deleting this generator we get an odd permutation and hence the result is different from $e$. Hence $g \notin A\left(S_{n}, \mathbf{g}\right)$.

2) Let $n=2 k$.

We set $\mathbf{g}=\{(1,2), \ldots,(n-1, n)\}$. The statement is clear for $n=2$. For $n=2 k>2$ the same arguments as in the previous case imply $A\left(S_{n}, \mathbf{g}\right) \neq\{e\}$. Let us show that $(1,2) \notin A\left(S_{n}, \mathbf{g}\right)$. Using the same parity arguments as in the odd case, we are left only with the case, when $(1,2)$ is written as a product of our generators, each occurring odd number of times. Let us delete $(2,3)$. Then $(1,2)$ will commute with all other generators left, and hence our product reduces to $(1,2)^{l} x$, where $x$ is odd and $x$ stabilizes both 1 and 2. Obviously $(1,2)^{l} x \neq 0$, a contradiction.

Any irreducible systems $\mathfrak{g}$ of generators of $S_{n}$, consisting of $n-1$ transpositions, corresponds to a tree with vertexes $\{1,2, \ldots, n\}$. If this tree has a fragment,

$$
i-j-k \cdots \cdots \cdots \cdots,
$$

where both $i$ and $j$ are not connected with any other elements, then the arguments, used in Example 24, imply $A\left(S_{n}, \mathfrak{g}\right)=A_{n}$. A natural question is: Is it true that $A\left(S_{n}, \mathfrak{g}\right)=S_{n}$ otherwise?

The previous consideration also motivates the following question: Let $G$ be a group and $\mathbf{g}=\left\{g_{1}, \ldots, g_{k}\right\}$ be a fixed system of generators in $G$. Let $F_{k}$ be the free group, generated by $\mathbf{x}=\left\{x_{1}, \ldots, x_{k}\right\}$, and $\varphi: F_{k} \rightarrow G$ be the epimorphism sending $x_{i}$ to $g_{i}$. Let $M$ be the kernel of $\varphi$ and $N$ be the normal subgroup of $F_{k}$, generated by $A\left(F_{k}, \mathbf{x}\right)$ and $M$. It is easy to see that $\varphi(N) \subset A(G, \mathbf{g})$. Is $\varphi(N)=A(G, \mathbf{g})$ ?

We finish the section with a description of the subgroup of almost identity elements in the group $U\left(n, \mathbb{Z}_{m}\right), m=\infty, 2,3,4, \ldots$, of unitriangular matrices.

Example 25. Let $U\left(n, \mathbb{Z}_{m}\right)$ be the group of unitriangular matrices $A=\left(a_{i, j}\right), i, j=$ $1, \ldots, n$, with coefficients in the ring $\mathbb{Z}_{m}$, i.e. $a_{i, j}=0, i>j ; a_{i, i}=1$, and $a_{i, j} \in \mathbb{Z}_{m}$. For $i=1, \ldots, n-1$ let $N(i) \in U\left(n, \mathbb{Z}_{m}\right)$ be such that $N(i)_{i, i+1}=1$ and $N(i)_{i, j}=0$ for all other $i \neq j$. It is known, see for example [R], that $\mathbf{N}=\{N(1), \ldots, N(n-1)\}$ is a system 
of generators for the nilpotent group $U\left(n, \mathbb{Z}_{m}\right)$. We claim that the following statements for $M \in U\left(n, \mathbb{Z}_{m}\right)$ are equivalent:

(1) $M \in A\left(U\left(n, \mathbb{Z}_{m}\right), \mathbf{N}\right)$.

(2) $M \in Z\left(U\left(n, \mathbb{Z}_{m}\right)\right)$, i.e. $M_{i, j}=0$ for all $i \neq j$ different from $(1, n)$.

(3) There is a word, $w=w(N(1), \ldots, N(n-1))$, such that $w=M$ and $\mathfrak{d}_{i}(w)=e$ for $i=1, n-1$.

Proof. (1) implies (3) by the definition of almost identity elements. The word

$$
w=[\ldots[[N(1), N(2)], N(3)] \ldots, N(n-1)]
$$

represents a generator of $Z\left(U\left(n, \mathbb{Z}_{m}\right)\right)$ and is obviously an almost identity word. Hence (2) implies (1). Therefore, to complete the proof it is enough to show that (3) implies (2).

Let $M \in U\left(n, \mathbb{Z}_{m}\right), M=w(N(1), \ldots, N(n-1))$, and $v=\left(v_{1}, \ldots, v_{n}\right) \in \mathbb{Z}_{m}^{n}$ be such that $v_{n}=0$. Then, since $\left\{\left(x_{1}, \ldots, x_{n-1}, 0\right) \mid x_{i} \in \mathbb{Z}_{m}\right\}$ is invariant under $U\left(n, \mathbb{Z}_{m}\right)$ and is pointwise stabilized by $N(n-1)$, it follows that $M v=\mathfrak{d}_{n-1}(w(N(1), \ldots, N(n-1)) v$. The last implies that if $w=w\left(N(1), \ldots, N(n-1)\right.$ satisfies $\mathfrak{d}_{n-1}(w)=e$, we necessarily have $M_{i, j}=0$ for all $j<n$ and $i \neq j$. Acting by $M$ on vectors in $\mathbb{Z}_{m}^{n}$ from the right we obtain that for $w$ satisfying $\mathfrak{d}_{1}(w)=e$ we necessarily have $M_{i, j}=0$ for all $i>1$ and $i \neq j$. This proves that the conditions in (3) imply (2), and completes the proof.

\section{Pulling back the faithful action to the quotient}

4.1. Our main motivation to study almost identity elements in free products of groups is the following construction of the action of the quotient modulo such elements. Let $G_{1}, \ldots, G_{k}$ be groups and $G=G_{1} * \cdots * G_{k}$.

Theorem 26. 1. If $G$ acts faithfully on $\mathcal{T}_{n}$, then $G / A\left(G_{1}, \ldots, G_{k}\right)$ acts faithfully on $\mathcal{T}_{n}$.

2. If $G$ acts faithfully on $\mathcal{T}_{n}$ by finite state automorphisms, then $G / A\left(G_{1}, \ldots, G_{k}\right)$ acts faithfully on $\mathcal{T}_{n}$ by finite state automorphisms.

Proof. Let $\varphi: G \hookrightarrow$ Aut $\mathcal{T}_{n}$ be a monomorphism and $f_{i}: G_{i} \hookrightarrow G, i=1, \ldots, k$, be canonical inclusions. We are going to define a new homomorphism, $\psi: G \rightarrow$ Aut $\mathcal{T}_{n}$, whose kernel will be precisely $A\left(G_{1}, \ldots, G_{k}\right)$. Since $G$ is a free product of $G_{i}$ it is enough to define $\psi$ on $f_{i}\left(G_{i}\right), i=1, \ldots, k$. Let us fix $i \in 1, \ldots, k$ and $g \in G_{i}$. We define the element $\psi\left(f_{i}(g)\right)$ using the following recursion: $\psi\left(f_{i}(g)\right)=\left[\alpha_{1}, e, \ldots, e, \beta_{1}\right]$, where

$$
\beta_{j}=\left\{\begin{array}{ll}
\varphi\left(f_{i}(g)\right) & j<k, j \neq i ; \\
e & \text { otherwise; }
\end{array} \quad \alpha_{i}=\left[\alpha_{i+1}, e, \ldots, e, \beta_{i+1}\right] .\right.
$$


Assume that we have chosen some generators in each $G_{i}$. Let $g \in G$ and $w$ be a word, representing $g$. By construction it is easy to see that on the level $i$ the element $\psi(g)$ acts as the identity on all branches except one, where it acts as $\mathfrak{d}_{i}(w)$. Since $\varphi$ is faithful, it follows that $\psi(g)=e$ if and only if $\mathfrak{d}_{i}(w)=e$ for all $i$, that is, if and only if $g \in A\left(G_{1}, \ldots, G_{k}\right)$. It is also clear that $\psi$ is finite automatic provided that $\varphi$ is so. This completes the proof.

The most natural case, where the notion of almost identity elements leads to nice combinatorics, is the case when all $G_{i}$ are cyclic groups of the same order. We consider the case of $\mathbb{Z}_{2}^{* k}=\mathbb{Z}_{2} * \cdots * \mathbb{Z}_{2}$ ( $k$ times). Fix a presentation, $\mathbb{Z}_{2}^{* k}=\left\langle a_{1}, \ldots, a_{k}\right| a_{i}^{2}=1, i=$ $1, \ldots, k\rangle$.

Remark 27. The group $N_{k}=\mathbb{Z}_{2}^{* k} / A\left(\mathbb{Z}_{2}^{* k},\left\{a_{1}, \ldots, a_{k}\right\}\right)$ has polynomial growth for $k=$ $1,2,3$ and exponential growth for $k>3$. Indeed, the statement is clear for $k=1,2$ as in this case already $\mathbb{Z}_{2}^{* k}$ has polynomial growth. For $k>3$ the group $N_{k}$ surjects onto $\mathbb{Z}_{2}^{*(k-1)}$ by Subsection 3.1. Since $\mathbb{Z}_{2}^{*(k-1)}, k>3$, is of exponential growth, so is $N_{k}$ in this case. Hence it is only left to study the case $k=3$. Write $\mathbb{Z}_{2}^{3}=\langle a\rangle *\langle b\rangle *\langle c\rangle$, where $a^{2}=b^{2}=c^{2}=e$ and define

$$
\varphi: \mathbb{Z}_{2}^{3} \rightarrow X=\langle a\rangle *\langle b\rangle \oplus\langle a\rangle *\langle c\rangle \oplus\langle b\rangle *\langle c\rangle
$$

via $\varphi(a)=(a, a, e), \varphi(b)=(b, e, b), \varphi(c)=(e, c, c)$. From the construction it follows immediately that $\varphi$ is a homomorphism with the kernel $A\left(\mathbb{Z}_{2}^{* k},\{a, b, c\}\right)$. Thus the image of $\varphi$ is isomorphic to a subgroup of $X$ and thus is of polynomial growth since $X$ is of polynomial growth.

The subgroup of almost identity elements in $\mathbb{Z}_{2} * \mathbb{Z}_{2}$ is just the commutator subgroup by Proposition 22 and hence is easy to describe. Example 27 shows that in the case $k=3$ the group $\mathbb{Z}_{2}^{* 3} / A\left(\mathbb{Z}_{2}^{* 3},\left\{a_{1}, a_{2}, a_{3}\right\}\right)$ seems to be rather special. Our attempt to give (so far in vain) a descent combinatorial description for the elements of $A\left(\mathbb{Z}_{2}^{* 3},\left\{a_{1}, a_{2}, a_{3}\right\}\right)$ has led to several nice combinatorial problems. One of them is the following: let $G=\langle a, b| a^{2}=$ $\left.b^{2}=e\right\rangle$, find the number $N$ of words of the form $a t_{1} a t_{2} \ldots a t_{2 m}$, where $t_{i}=e$ or $t_{i}=b$ for all $i$, which are equal to $e$ in $G$.

Proposition 28. $N=\left(\begin{array}{c}2 m-1 \\ m\end{array}\right)$.

Proof. Let $S(m, l)$, where $l$ is even, denote the set of all words of the form $a t_{1} a t_{2} \ldots a t_{2 m}$, where $t_{i}=e$ or $t_{i}=b$ for all $i$, which are equal to $(b a)^{l}$ in $G$; and $K(m, l)=|S(m, l)|$. It is clear that $K(1,0)=1, K(1, l)=0, l \neq 0$. For $m>0$ the map $x \mapsto x a a$ induces an injection, $\mathfrak{i}_{1}^{m, l}: S(m, l) \hookrightarrow S(m+1, l)$; and the map $x \mapsto x a b a b$ induces an injection, $\mathfrak{i}_{2}^{m, l}: S(m, l) \hookrightarrow S(m+1, l-2)$.

For $x \in S(m, l), x=a t_{1} a t_{2} \ldots a t_{2 m}$ we denote by $\overline{x b}$ the word $a t_{1} a t_{2} \ldots a t_{2 m-1} a b$ if $t_{2 m}=e$ and the word $a t_{1} a t_{2} \ldots a t_{2 m-1} a e$ otherwise. Using this notation the map $x \mapsto \overline{x b} a a b$ induces an injection, $\mathfrak{i}_{3}^{m, l}: S(m, l) \hookrightarrow S(m+1, l)$; and the map $x \mapsto \overline{x b} a b a$ induces an injection, $\mathfrak{i}_{4}^{m, l}: S(m, l) \hookrightarrow S(m+1, l+2)$. 


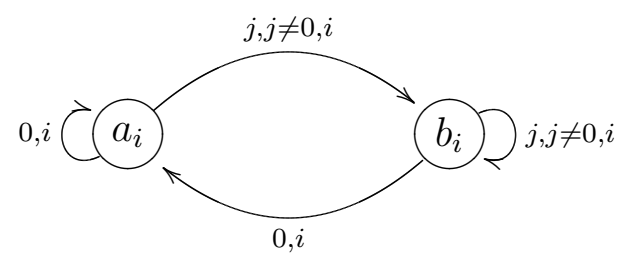

Figure 2: The automaton describing automorphisms $a_{i}$ and $b_{i}$

It follows from the definition that the images of $\mathfrak{i}_{1}^{m, l}, \mathfrak{i}_{2}^{m, l+2}, \mathfrak{i}_{3}^{m, l}$, and $\mathfrak{i}_{4}^{m, l-2}$ do not intersect and cover $S(m+1, l)$, which gives us the recursive relation

$$
K(m, l)=K(m-1, l-2)+2 K(m-1, l)+K(k-1, l+2),
$$

which is a known recursion for binomial coefficients. It follows that $K(m, l)=\left(\begin{array}{c}2 m-1 \\ m+l\end{array}\right)$ and $N=K(m, 0)=\left(\begin{array}{c}2 m-1 \\ m\end{array}\right)$.

Proposition 28 stimulates to ask: is there any natural bijection between the words described above and the m-element subsets in a $2 m$-1-element set.

4.2. Fix arbitrary integer $n \geq 2$. Denote by $\tau_{i}, 1 \leq i \leq n$, the transposition $(0, i)$ on the set $\{0,1, \ldots, n\}$. Define automorphisms $a_{1}, \ldots, a_{n}$ of the tree $\mathcal{T}_{n+1}$ as follows: for $1 \leq i \leq n$ let

$$
a_{i}=\left(c_{0}, c_{1}, \ldots, c_{n}\right) \tau_{i} \quad \text { and } \quad b_{i}=\left(c_{0}, c_{1}, \ldots, c_{n}\right),
$$

where for $0 \leq j \leq n$ we have

$$
c_{j}= \begin{cases}b_{i}, & j=0, \\ b_{i}, & j=i, \\ a_{i}, & \text { otherwise. }\end{cases}
$$

Both automorphisms $a_{i}$ and $b_{i}$ have only two different states, namely $a_{i}$ and $b_{i}$, and can be described by the automaton on the Figure 2. Denote by $G_{n}$ the subgroup of Aut $\mathcal{T}_{n+1}$, generated by automorphisms $a_{1}, \ldots, a_{n}$, and by $H_{n}$ the subgroup generated by $b_{1}, \ldots, b_{n}$.

Theorem 29. The groups $G_{n}$ and $H_{n}$ are isomorphic to the free product $\mathbb{Z}_{2}^{* n}$ of $n$ cyclic groups of order 2.

Proof. Consider the group $G_{n}$. For $H_{n}$ the proof is analogous. We have

$$
a_{i}^{2}=b_{i}^{2}=\left(c_{0}^{2}, c_{1}^{2}, \ldots, c_{n}^{2}\right)
$$

and hence $a_{i}^{2}=b_{i}^{2}=e$ which implies that both $a_{i}$ and $b_{i}$ have order 2 .

Let us call an automorphism, $a \in$ Aut $\mathcal{T}_{n+1}$, rooted essential if its rooted permutation is non-trivial and the rooted permutation of the first state of the $k$-th level of $a$ (where $k>0$ ) is trivial. In particular, if some automorphism $b$ has a rooted essential state then $b$ is nontrivial. 
Let us prove that a product, $a_{i_{1}} \ldots a_{i_{m}}, 1 \leq i_{1}, \ldots, i_{m} \leq n$, does not equal $e$ provided that $i_{j} \neq i_{j+1}, 1 \leq j \leq m-1$. In fact, we prove by induction on $m$ that the first state of the $(m-1)$-th level of this product is rooted essential with the rooted permutation $\tau_{i_{m}}$. In case $m=1$ the required assertion follows from the definition of $a_{i_{1}}$. Assume that the assertion is true for the product $a_{i_{1}} \ldots a_{i_{m-1}}$. Denote by $d$ the first state of the $(m-1)$-th level of this product and calculate the first state of the $(m-1)$-th level of $a_{i_{1}} \ldots a_{i_{m}}$. Since $i_{m-1} \neq i_{m}$, the latter state equals $d \cdot a_{i_{m}}$. Applying the inductive assumption and the definition of $a_{i_{m}}$ we obtain that this automorphism is rooted essential with rooted permutation $\tau_{i_{m}}$.

Hence the group $G_{n}$ is generated by $n$ involutions which admit no additional relations and the proof is complete.

Note that this is the minimal example of a representation of $\mathbb{Z}_{2}^{* n}$ by finite state automorphisms of a rooted tree as its generators have only 2 different states. There arises a natural question: Is it possible to construct such a representation for the tree $\mathcal{T}_{m}, m<n+1$, with generators having only 2 different states?

Acknowledgments. The results were obtained during the visits of the first, the third and the fourth authors to Uppsala University, and the second author to Kyiv University, which were supported by The Swedish Institute. The hospitality of the host Universities and financial support of The Swedish Institute are gratefully acknowledged. We would like to thank V.Nekrashevych for helpful discussions.

\section{References}

[Bi] J. Birman, Braids; Links and Mapping Class Groups, Ann. Math. Stud., Princeton University Press, 1975.

[BS] A. Brunner, S. Sidki, The generation of $\operatorname{GL}(n, \mathbb{Z})$ by finite state automata. Int. J. Algebra Comput. 8, (1998), No.1, 127-139.

[CP] J. Crisp, L. Paris, The solution to a conjecture of Tits on the subgroup generated by the squares of the generators of an Artin group. Invent. Math. 145 (2001), no. $1,19-36$.

[FR] M. Falk, R. Randell, Pure braid groups and products of free group. Contem. Math. 78 (1988), 217-228.

[Ga] F. Gantmacher, The Theory of Matrices, Vol. 2. Providence, RI: AMS Chelsea, 2000 .

[Gr] R. Grigorchuk, Just infinite branch groups, New horizons in pro-p-groups (Markus P. F. du Sautoy Dan Segal and Aner Shalev, eds.), Birkhèauser Boston, Boston, MA, 2000, pp. 121-179. 
[GNS] R. Grigorchuk, V. Nekrashevich, V. Sushchansky, Automata, dynamical systems, and groups. (Russian) Tr. Mat. Inst. Steklova 231 (2000), Din. Sist., Avtom. i Beskon. Gruppy, 134-214; translation in Proc. Steklov Inst. Math. 2000, no. 4 (231), 128-203

[MKS] W. Magnus, A. Karras, D. Solitar, Combinatorial group theory: Presentations of groups in terms of generators and relations. Interscience Publishers, New YorkLondon-Sydney. 1966 xii+444 pp.

[N] V. Nekrashevych, Self-similar groups, volume 117 of Mathematical Surveys and Monographs. Amer. Math. Soc., Providence, RI, 2005.

[Ol1] A. Olijnyk, Free products of $C_{2}$ as groups of finitely automatic permutations, Voprosy Algebry, v. 14 (1999), 158-165.

[Ol2] A. Olijnyk, Free products of finite groups and groups of finitely automatic permutations, Proc. Steklov Inst. Math. 2000, no. 4 (231), 308-315.

[R] D. Robinson, A course in the theory of groups. Springer-Verlag, New York-BerlinHeidelberg. 1993.

[S] S. Sidki, Regular Trees and their Automorphisms. Momografias de Matematica, vol. 56, IMPA, Rio de Janeiro, 1998.

Yaroslav Lavrenyuk, Department of Mechanics and Mathematics, Kyiv Taras Shevchenko University, 64, Volodymyrska st., 01033, Kyiv, Ukraine,

e-mail: ylavrenyuk@univ.kiev.ua

Volodymyr Mazorchuk, Department of Mathematics, Uppsala University, Box 480, 751 06, Uppsala, Sweden, e-mail: mazor@math.uu.se, web: "http://www.math.uu.se/mazor/".

Andriy Oliynyk, Department of Mechanics and Mathematics, Kyiv Taras Shevchenko University, 64, Volodymyrska st., 01033, Kyiv, Ukraine, e-mail: olijnyk@univ.kiev.ua

Vitaliy Sushchansky, Institut of Mathematics, Silesian University of Technology, 23, ul Kaszubska, 44-100, Gliwice, Poland, email: Wital.Suszczanski@polsl.pl

and Department of Mechanics and Mathematics, Kyiv Taras Shevchenko University, 64, Volodymyrska st., 01033, Kyiv, Ukraine, e-mail: wsusz@univ.kiev.ua 